\title{
Interactive Illustration of Collage for Children with Folktale E-book
}

\author{
Dongwann Kang • Feng Tian • Reza \\ Sahandi
}

Received: date / Accepted: date

\begin{abstract}
It is always challenging to teach children foreign languages, due to the difficulty of learning and their short attention span. To address the challenge and take advantage of the popularity of touchable tablets and smartphones, we propose an educational folktale e-book (EFE-Book) application with an interactive illustratable tool. EFE-Book is developed to teach preschool children to learn foreign languages by telling folktales with illustrations. To encourage effective learning, EFE-Book provides an interactive collage tool that enables users to create collage-based illustrations by hand. We propose a Voronoi diagram based approach to model paper tiles for the development of EFE-Book. With EFE-Book, the user can create coloured paper tiles and attach them to the predesigned sketch through touch interface, such as Apple iPad.
\end{abstract}

Keywords mobile $\cdot$ e-learning $\cdot$ e-book $\cdot$ collage $\cdot$ stylization

\footnotetext{
Dongwann Kang

Faculty of Science \& Technology, Bournemouth University

Fern Barrow, Poole, Dorset, BH12 5BB, United Kingdom

Feng Tian

Faculty of Science \& Technology, Bournemouth University Fern Barrow, Poole, Dorset, BH12 5BB, United Kingdom Tel.: +44-1202-965250

E-mail: ftian@bournemouth.ac.uk

Reza Sahandi

Faculty of Science \& Technology, Bournemouth University Fern Barrow, Poole, Dorset, BH12 5BB, United Kingdom
} 


\section{Introduction}

Many mobile applications in various fields such as games, e-books and elearning have been developed in recent years, thanks to the advancement of mobile technology. Touchable screens are now widely used for display devices such as smartphones and tablet PCs, making interaction between users and applications much easier. Many drawing tools are now available through mobile applications, for example converting a photo into an image with artistic styles $[2,8]$.

For educational applications, the interaction and motivational elements are the most important, especially when the target users are children. In this sense, the stylization techniques with interaction can be an effective part of educational applications. So in this paper, we propose an educational folktale e-book (EFE-Book) for preschool children to learn foreign languages. Using the collage art technique, the EFE-Book provides an interactive illustratable tool and makes learning more effective and enjoyable.

The paper is organized as follows. Section 2, provides an overview of related work in mobile applications for e-learning and collage style rendering. Section 3 provides details of the EFE-Book with interactive illustratable tools. The results are discussed in Section 4. Finally, conclusion with a summary of our ideas and our future plans for further developments are discussed in Section 5 .

\section{Related Work}

Mobile devices have been considered as a good supplementary to support both students and teachers by providing services that facilitate teaching, learning and education related administrative tasks, e-book and e-learning [10]. Jacott [7] explained applications and effectiveness of mobile learning, which provides useful, authoritative and comprehensive guidance for professionals in education. With its wide use and popularity, the smartphone has become a powerful mobile device for e-learning and e-book. In Godwin-Jones's article [4], he explored the state of language learning applications, devices they run on, and how they are developed. Especially for language learning, Mori et al. [12] proposed an interactive e-book application on smartphone. Their application was based on a conversational agent with animated character that asks questions or makes comments about the current page. These features made the book significantly more interactive and engaging when compared to static e-books. Compared with our application, the application of Mori et al. mainly focuses on the assist of learning, while our application focuses on motivating learning.

The collage stylization researches based on coloured paper have mainly focused on generating coloured paper tiles and placing them appropriately. Seo et al. [14] proposed a method that converts an input image into a coloured paper mosaic. In their method, the coloured paper tiles are generated by using the Voronoi diagrams [1]. To replicate the torn paper effect, they ap- 
plied the random midpoint displacement method. Gi et al. [3] proposed a torn simulation-based coloured paper mosaic method. The method tears off paper tiles from given coloured papers, and places them on the most appropriate locations in an image. To locate tiles and minimize gaps and overlaps between tiles, they also proposed an energy function to avoid crossing edges in the image. By minimizing the energy function, a realistic coloured paper mosaic image is generated. In contrast, Han et al. [5] focused on the sequence in which tiles are placed, and proposed an approach that sequentially places each tile according to a customizable rule defined by parameters. This method was the first to enable placement of tiles along an edge, or to place tiles that have a similar color to previous tiles, by adjusting the parameters. In this paper we partially employ some of the main ideas of the coloured paper tile stylization proposed in the above studies.

Our preliminary studywas published in [9]. In this paper we have developed a new exam functionforEFE-Book to enhancelearning, andimproved theillustratabletoolwith an assistance function. EFE-Book has also been expanded with a library which consists of a number of e-books with separate stories and illustrations. Moreover, we provide more experimental results which show that the illustratable tool in our application is effective for improving learning.

\section{Development of EFE-Book with Interactive Illustratable Tool for Collage Art}

In this section we give an overview of interfaces and functions of our e-book application for foreign language education, and propose an illustratable tool for collage art on the e-book.

\subsection{The EFE-Book for preschool kids' learning foreign languages}

\subsubsection{Library}

EFE-Book provides a library which consists of a number of e-books with separate stories and illustrations. When EFE-Book starts, it requires user to login to a server to access the library, as shown in Figure 1(a). If a user's account is validated, the server sends the Content Delivery Network address to our EFEBook, which then downloads encrypted data of each book from the address, and loads them onto the library (Figure 1(b)). When the user selects a book on the library, a brief story with illustration is displayed as seen in Figure 1(c). Then the user can start reading and learning the book.

\subsubsection{The EFE-Book}

An overview of the EFE-Book is presented in Figure 2. It consists of a cover page, several scenes, and exam page. Since too many scenes may shorten the 


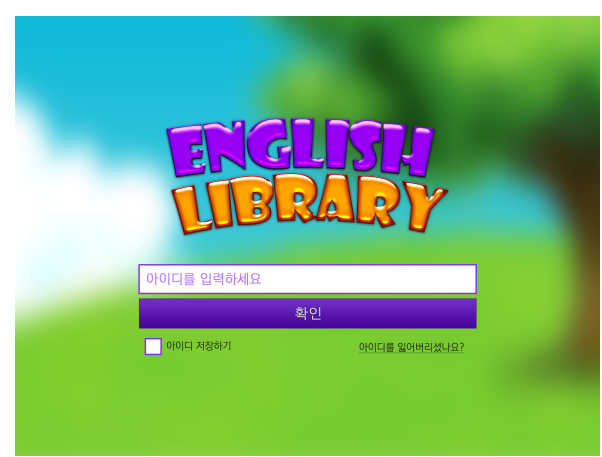

(a) Log-on display

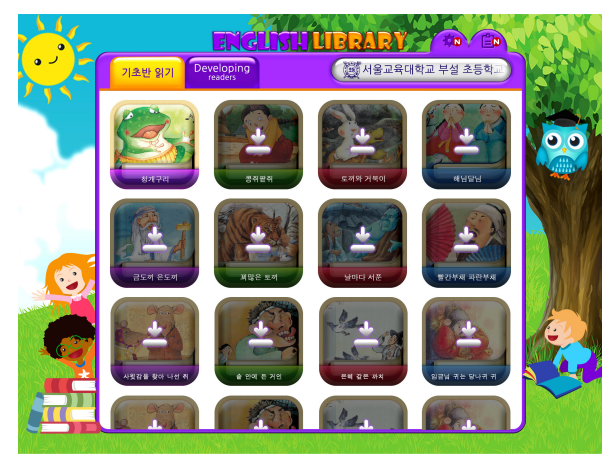

(b) The library of EFE-Books

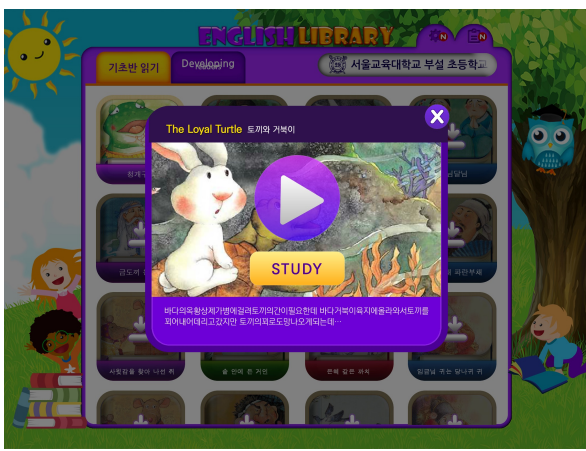

(c) An example of selected e-book

Fig. 1 Our application provides a library which allows access to various EFE-Books. This requires appropriate validation from server through log-on step.

span of pre-school children, we limit the length of the scenes to 10. Each scene shows a representative illustration with a script which is a tale describing the scene. Figure 3 represents the layers of each scene. On the top layer, a menu bar and navigator buttons are located on the bottom and both sides of the scene, respectively. With navigator buttons, user can move to previous or next scene. The menu bar consists of several functions including replay 


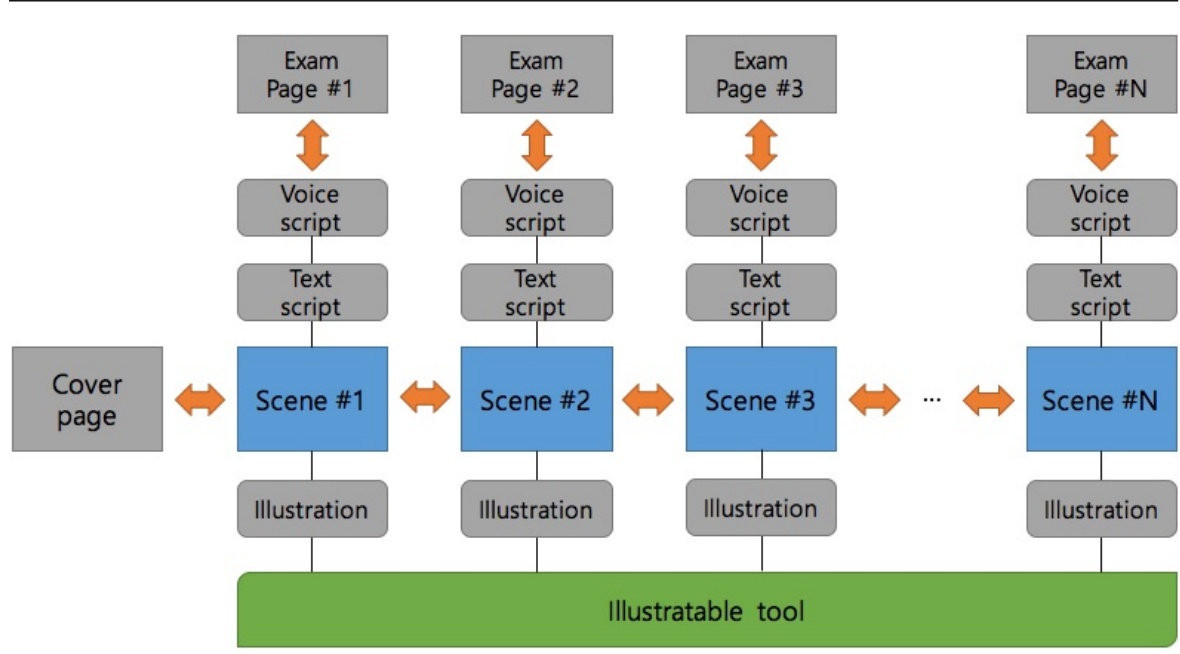

Fig. 2 Overview of the EFE-Book. The application consists of illustrated scenes with a short script. In each scene, a script is visually and auditorily played with the illustration. With a menu on each scene, user can interactively create illustration through the proposed tool for collage art.

which restarts the current scene, a table of contents that allows moving to a specific scene, an illustratable tool (more details in Section 3.2), and a movie function that plays all the scenes continuously. On the middle layer, a script is placed on a side of a scene. When a user enters a scene, a script is popped up. Then, a voice script is played with background music. At this time, the user may turn off the voice script to concentrate on reading. Moreover, the script can be replaced with another language using a language toggle button on the upper right corner of the scene. The EFE-Book provides scripts (both text and voice) with two languages, English and Korean. On the bottom layer, the illustration fills the entire scene. The illustrations in the EFE-Book are captured from original tale books, so they are static. However, we utilize fade in/out and zoom in/out effects to make the scenes dynamic when user enters each scene. At each scene, the user can create his/her own unique illustration with collage art through an illustratable tool from the menu. The detail of the illustratable tool is described in the next section.

\subsubsection{Exam page}

After users finish reading each page of an e-book, our EFE-Book presents them an exam page which contains a multiple choice question in foreign language, as shown in Figure 4. Because children have short attention span, we provide only one question per page. The questions are classified into two types: one is to choose an appropriate word for the blank space of a sentence presented in the book; and the other is to select a proper word for a given meaning which is described in native language (here Korean). After user selects an answer for each question, a correct answer is showed to tell user whether he/she has 


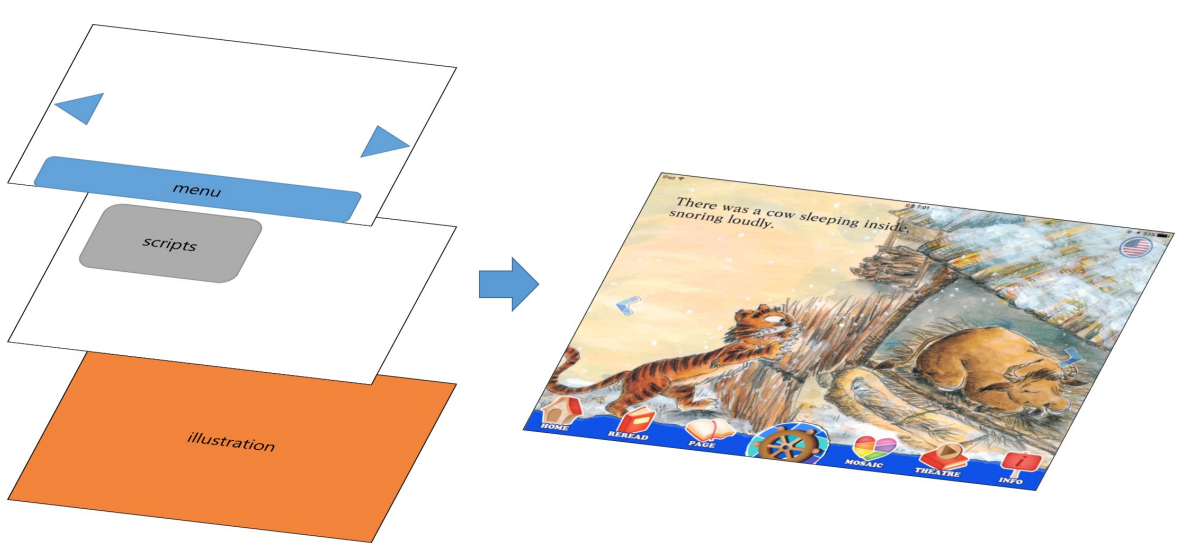

Fig. 3 Three layers of scenes. Menu bar and navigator buttons are located on the top layer; script is located on the middle; and illustration is located on the bottom.

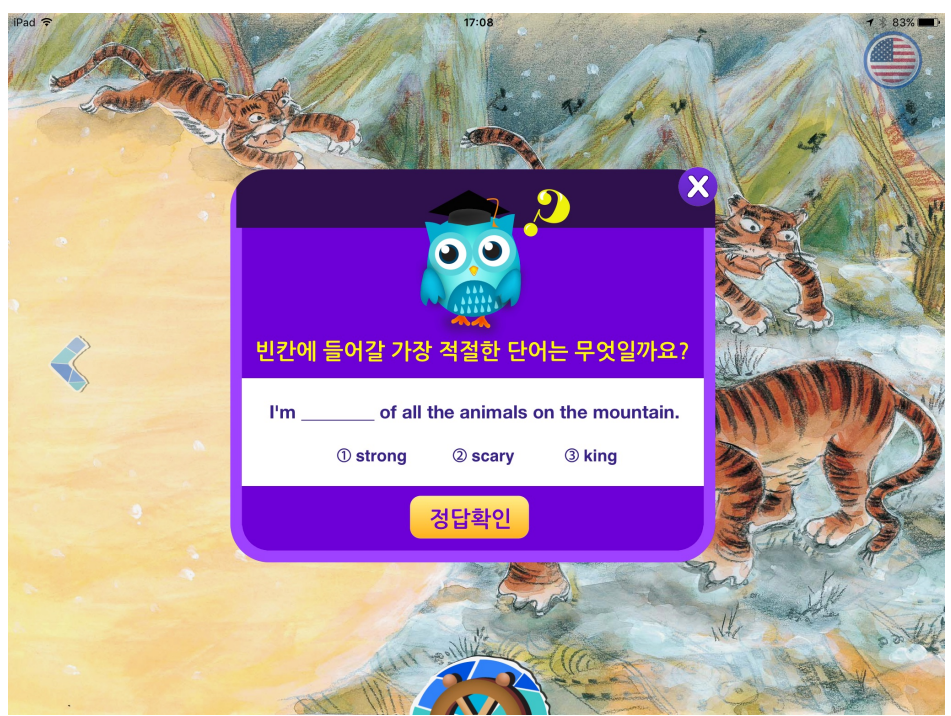

Fig. 4 Exam page. After reading each page, users are provided a question to test their study in foreign language.

got it correct. When all questions are finished through an e-book, the total score is displayed. At this time, EFE-Book records user's last score into user's device, so that the user can compare their current score with the previous ones. Note that, to prevent user from being tested with same questions every time, EFE-Book randomly sample 10 questions from its database of exam questions. 

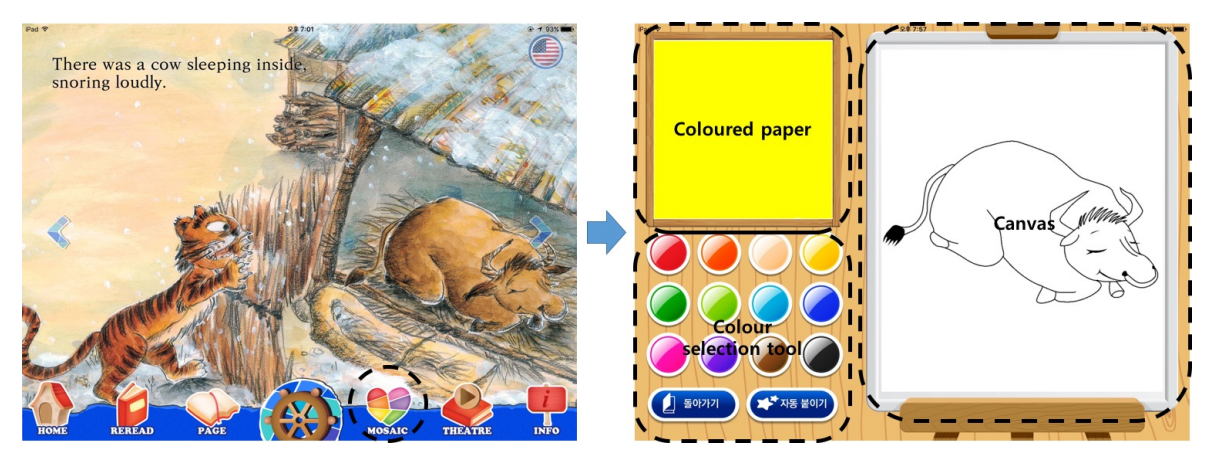

Fig. 5 Interactive illustratable mode

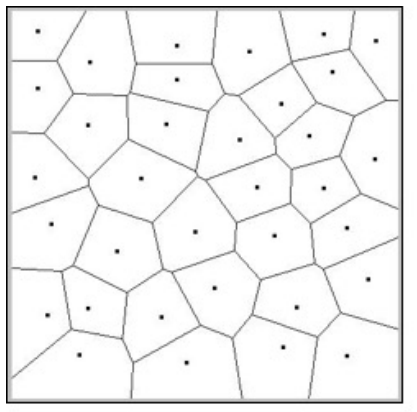

Initial Voronoi diagram

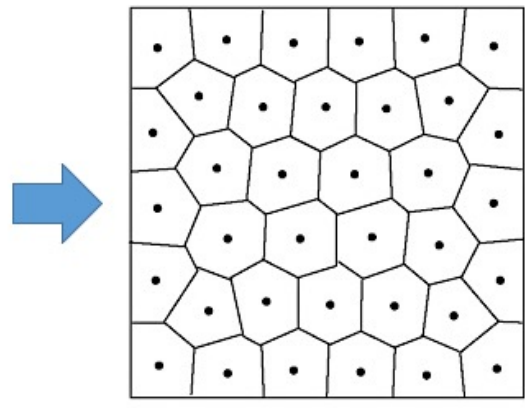

After Lloyd's relaxation

Fig. 6 Dividing coloured paper into small tiles with the Voronoi diagram and the Lloyd's relaxation method. Each cell of the Voronoi diagram is used to create coloured paper tiles.

\subsection{Interactive illustratable tool for collage art}

When a user selects the illustratable tool from the menu bar, the screen changes into the interactive illustratable mode, as shown in Figure 5. The screen in this mode consists of two panels; a white canvas with a sketch on the right hand side panel, and a coloured paper including a colour selection tool on the left hand panel. On the canvas, the predesigned sketch of an illustrated character in is located. The object is not filled with colours, so that user can fill it with coloured paper tiles. When user touches the coloured paper on the left hand panel and drags it to canvas, a small coloured paper tile is torn out from the coloured paper, and moves along to the trajectory of touched finger. Subsequently, the user can attach it to appropriate position on the canvas by keeping dragging. The colour selection tool may be used for changing colours.

\subsubsection{Modeling coloured paper tiles}

When user enters the interactive illustratable mode, the coloured paper on the left panel is pre-divided into many small tiles. To divide the coloured paper 
into tiles, we employ the Voronoi diagram [6] as shown in Figure 6. A Voronoi cell $C_{i}$ is defined by using its pixel $c$, as shown in following equation.

$$
C_{i}=\left\{c \in C_{i} \mid D\left(c, S_{i}\right)<D\left(c, S_{j}\right)\right\} \text { for all } j,
$$

where $S_{i}$ is the site of $C_{i}$ and $D$ is a Euclidean distance function.

For rectangular coloured paper on the left hand panel, we generate the initial Voronoi diagram by randomly inserting seed points, and relax the distribution of the seed points by using the Lloyd's method [11] to obtain uniform sized Voronoi cells. The coloured paper tiles are then created by converting each cell into polygon and filling them with selected colours. Although the coloured paper is divided into tiles, this is not visible on the panel, as each tile is seamlessly positioned without any gap between tiles.

\subsubsection{Simulating torn paper effect}

In the coloured paper collage art, each tile has unique torn paper effects on their edges. We employ the random midpoint displacement method to simulate the torn effect. On each tile's edge, we select a midpoint, and subdivide the edge into two segments on that midpoint. The midpoint is then randomly displaced along a direction perpendicular to the edge. This is achieved by applying the following equation which calculates the randomly displaced midpoint:

$$
\begin{aligned}
& f\left(p_{1}, p 2\right)=\frac{1}{2}\left(p_{1}+p_{2}\right)+r v_{p} \\
& v_{p}=R_{90} \overrightarrow{p_{1} p_{2}}, R_{90}=\left[\begin{array}{cc}
0 & -1 \\
1 & 0
\end{array}\right]
\end{aligned}
$$

where $p_{1}$ and $p_{2}$ are the points on both sides of an edge and $r$ is a random value within $[-t: t]$ which adjusts the range of displacement offset. In this study, we use $t=0.25$ as the value of $t$. Until the subdivided segments reach third iteration, we perform these steps iteratively. This approach generates an irregular torn shape on the edge of tile as shown in Figure 7. However, in real torn paper, we can observe that the white tissue of paper is revealed. To mimic this effect, under the original Voronoi cell of the tile, we add another white Voronoi polygon which has the same shape with the original cell, and apply the random midpoint displacement algorithm separately. As a result, we can get a torn paper effect similar to that in real torn paper as shown in Figure 8. When user starts dragging tiles from the coloured paper on the left panel, a torn paper effect is added to the edge of the tile.

\subsubsection{Assistance of locating paper tile}

To assist users to locate paper tiles easily onto appropriate position, EFEBook provides an assistance function. When a user drags a tile from coloured paper panel to canvas, the function indicates proper positions of current tile. To implement this function, we employed the position-based tiling algorithm 


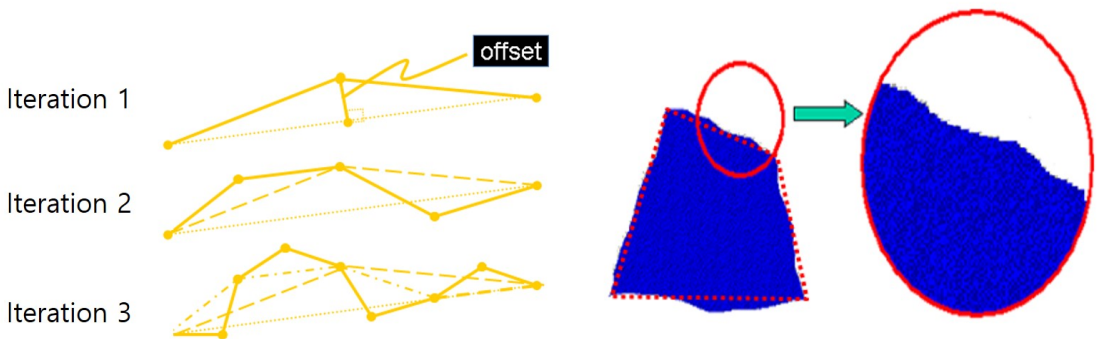

Fig. 7 Random midpoint displacement algorithm for torn paper effect.
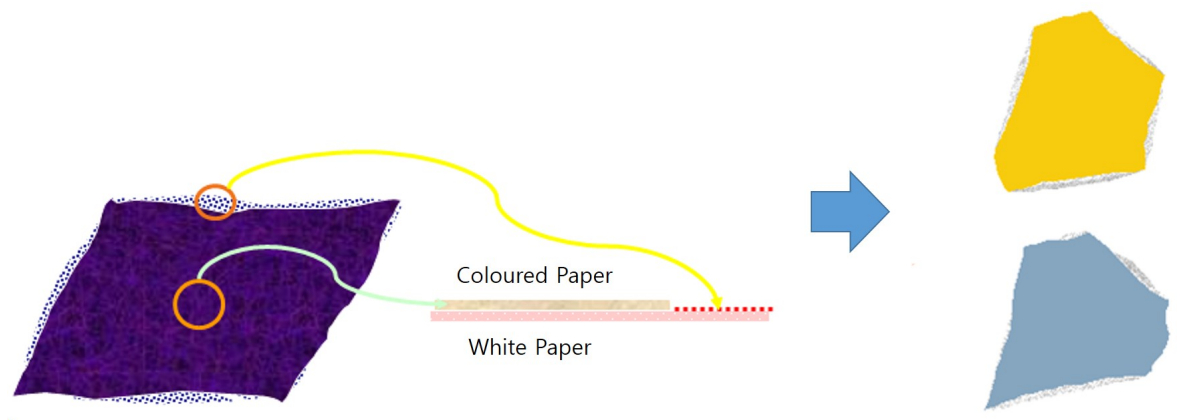

Fig. 8 Representation of the realistic torn paper effect. An additional white paper is added under the tile, and the random midpoint displacement algorithm is applied separately.

[5] which finds appropriate locations of tiles by considering the edges of the image and the shapes of neighbor tiles. Following the algorithm, we generate offset lines from the edge of sketch and the boundaries of pre-located tiles, and find the best two positions of current tile among the points on the offset lines. As shown in Figure 9, according to the color and shape of dragging tile, the best two appropriate positions are displayed on the canvas, so that user can easily complete the collage.

\section{Results}

We implemented EFE-Book by using OpenGL ES 2.0 [13] on Apple iPad's iOS environment, as shown in Figure 10.

Figure 11 shows each step of illustration. User can navigate between scenes, and study a folktale with scripts written in a couple of languages including users' native language. Both text scripts and voice scripts are presented or played on each scene, so that user can learn the correct pronunciation of the foreign language such as English. At each scene, user can create illustrations through the illustratable tool. By touching and dragging tiles, the illustration of the paper collage art style can be generated. After the user finishes making collages, the result is displayed on the original scene of the EFE-Book, as 

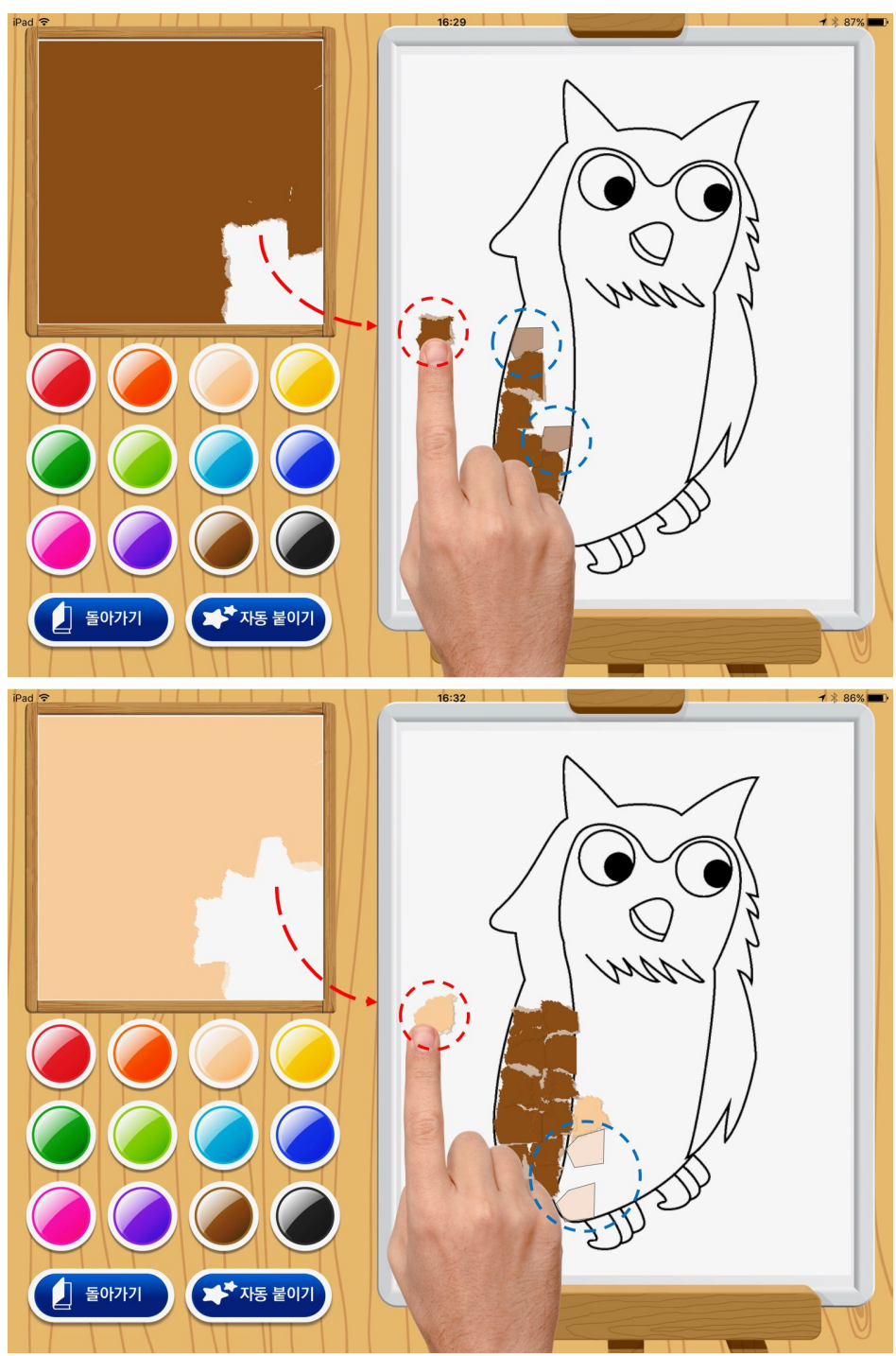

Fig. 9 Tile location assistance function. When user drags a paper tile (red circle) from the coloured paper panel, two of appropriate locations (blue circle) appear to assist locating the tile.

shown in the Figure 11, giving the impression that the user has created the illustration of the book. Figure 12 shows various collage results generated with the EFE-Book.

At the end of the application, we provided a link of webpage for a survey, as illustrated in Figure 13(a), to evaluate EFE-Book's usability and usefulness. For this survey, we asked the parents of children to answer a couple of enquiries about the usability and usefulness. In total 32 users have responded. For the 


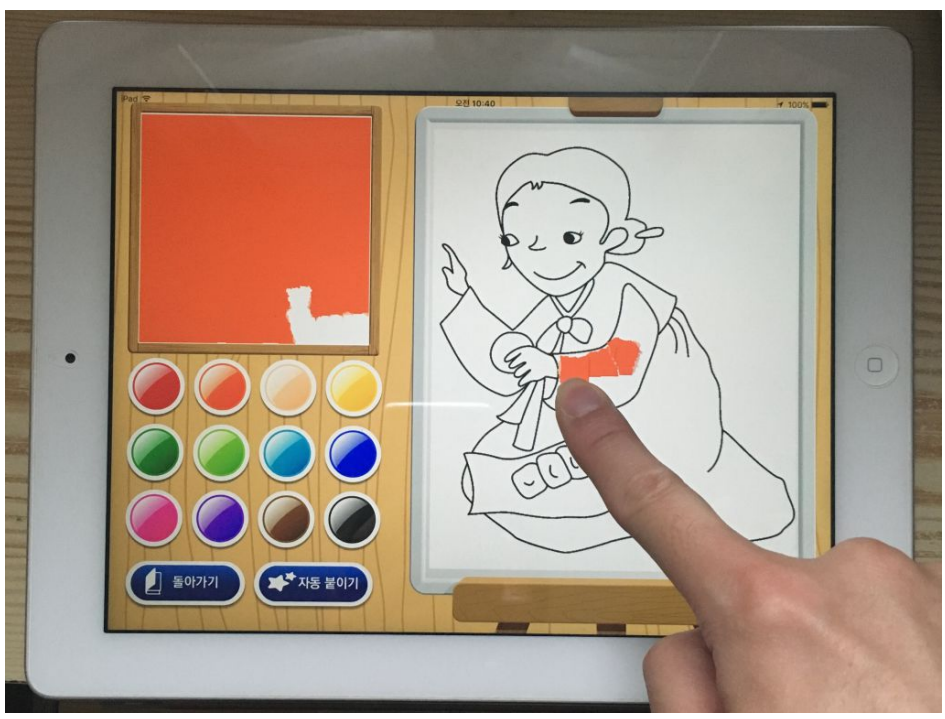

Fig. 10 EFE-Book on Apple iPad.

usability, we asked users how easy to use our application was. As shown in Figure 13(b), most of users responded that our application was relatively easy to use. According to users' comments on the survey, most children were very interested in doing collage with the tool, so that they read our EFE-Book in order to perform collage. There were a few minor comments about usability. However, because the size of tile was constant, it was difficult to locate tiles on narrow region. To overcome this, we will provide tile size control module in future version. For the usefulness of the tool, the question is how well our interactive illustratable tool on the EFE-Book helps in attracting children for studying foreign languages. As shown in Figure 13(c), most of users agreed that the tool was useful for children's learning.

To evaluate the effectiveness of our illustratable tool for learning foreign language, we compared users' initial score (of the exam provided in EFE-Book) with the score which was recorded two weeks later. To do so, we first recruited 20 users who have 7-8 years old children from online community. Then, we provided them our EFE-Book with same content. At this time, we divided users into two groups; one consists of 10 users who have EFE-Book with full functions (Group A in Table 1), and the other who also consists of 10 users was provided with another version of EFE-Book in which the illustratable tool is removed (Group B in Table 1). We asked them to let their children to do exams during first reading, and the results were collected to our server. Two weeks later, we asked them again, and compared the results with the previous one. As shown in the Table 1, a user group with the illustratable tool achieved $36.8 \%$ of improved rate while the other group without the illustratable tool did $17.7 \%$. Thus we could conclude that the illustratable tool is successfully to encourage users to learn foreign language. 


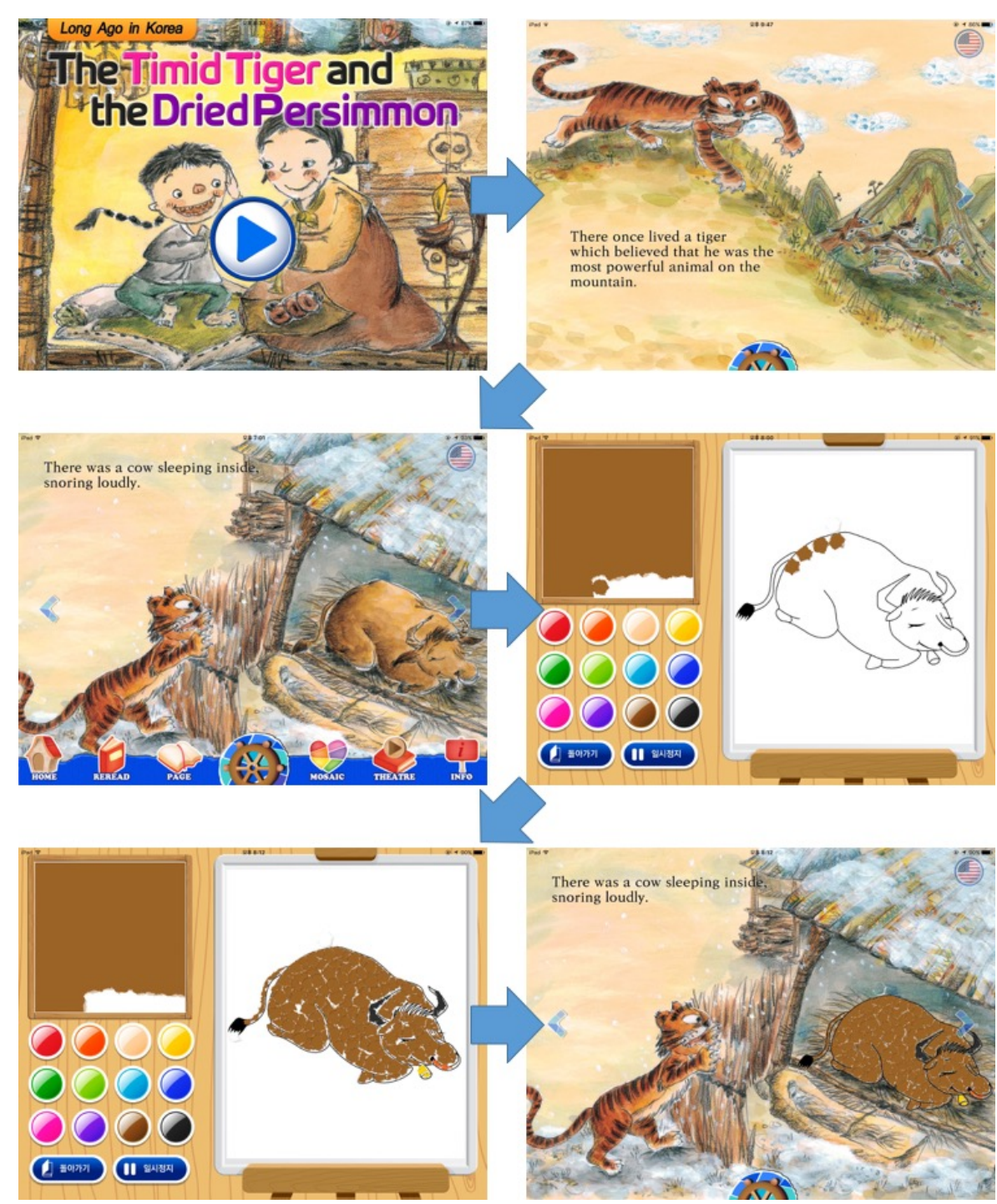

Fig. 11 Procedure of EFE-Book. While reading and listening to the book, user can create illustration through our interactive illustratable tool.

\section{Conclusion}

In this paper we discussed our proposed e-book application (EFF-Book) to teach pre-school children foreign languages using devices such as smartphones or iPads. By utilizing the touch screen, our application provides an interactive illustratable tool for collage art. With folktale contents, our proposed illustratable tool can encourage the pre-school children to concentrate more while learning. 


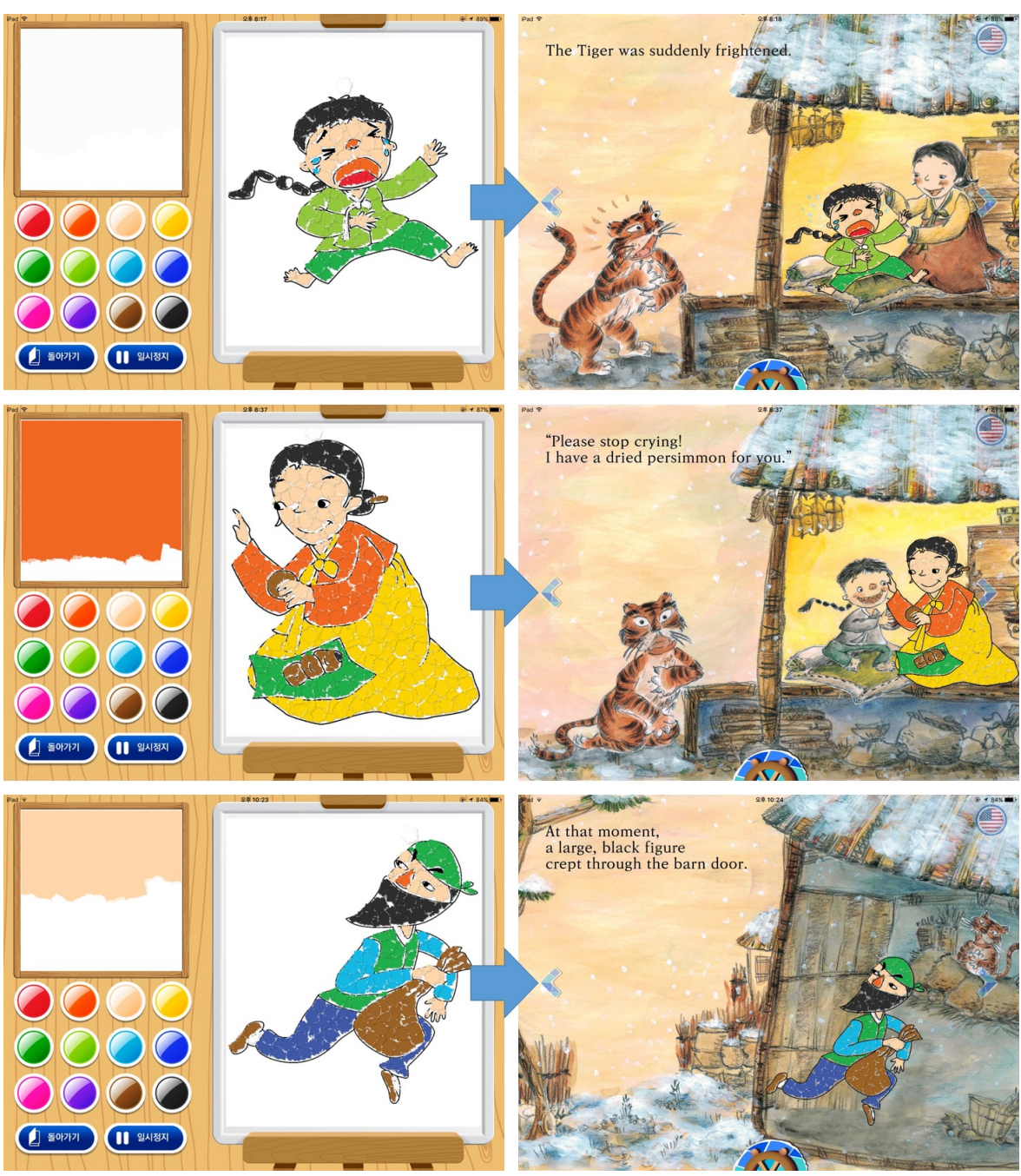

Fig. 12 Results generated with the EFE-Book. After the user finishes making collages, the result is displayed on the original scene of EFE-Book.

As mentioned in Section 4, our collage illustrative tool provides only same sized tiles, so that tiles occasionally cannot fit narrow regions. To tackle this, we will develop tile size control module in future version. In addition, this work will be extended to other styles such as paintings, drawings, etc. For this, we will study simulation of various styles with an intuitive interface.

Acknowledgements This research was supported by Basic Science Research Program through the National Research Foundation of Korea (NRF) funded by the Ministry of Education (NRF-2016R1A6A3A03010386). 
Table 1 Evaluation for the effectiveness of our illustratable tool.

\begin{tabular}{|c|c|c|c|c|c|c|c|c|c|c|c|c|c|c|c|c|c|c|c|c|c|c|c|c|}
\hline \multirow[b]{2}{*}{ user id } & \multicolumn{12}{|c|}{ Group A (w/ illustratable tool) } & \multicolumn{12}{|c|}{ Group B (w/o illustratable tool) } \\
\hline & $\mathrm{a}$ & $\mathrm{b}$ & $\mathrm{c}$ & $\mathrm{d}$ & $\mathrm{e}$ & $\mathrm{f}$ & $\mathrm{g}$ & $\mathrm{h}$ & $\mathrm{i}$ & $\mathrm{j}$ & st.dev. & avg. & $\mathrm{k}$ & 1 & $\mathrm{~m}$ & $\mathrm{n}$ & $\mathrm{o}$ & $\mathrm{p}$ & $\mathrm{q}$ & $\mathrm{r}$ & $\mathrm{s}$ & $\mathrm{t}$ & st.dev. & avg. \\
\hline Init. Score & 8 & 4 & 5 & 6 & 4 & 5 & 6 & 7 & 6 & 6 & 1.25 & 5.7 & 5 & 5 & 6 & 7 & 4 & 5 & 8 & 9 & 6 & 7 & 1.55 & 6.2 \\
\hline Final score & 10 & 8 & 6 & 8 & 7 & 8 & 7 & 7 & 8 & 9 & 1.14 & 7.8 & 4 & 7 & 7 & 9 & 7 & 6 & 7 & 10 & 8 & 8 & 1.64 & 7.3 \\
\hline$+/-$ & 2 & 4 & 1 & 2 & 3 & 3 & 1 & 0 & 2 & 3 & 1.20 & 2.1 & -1 & 2 & 1 & 2 & 3 & 1 & -1 & 1 & 2 & 1 & 1.29 & 1.1 \\
\hline
\end{tabular}

\section{References}

1. Aurenhammer, F.: Voronoi diagrams\&mdash;a survey of a fundamental geometric data structure. ACM Comput. Surv. 23(3), 345-405 (1991). DOI 10.1145/116873.116880. URL http://doi.acm.org/10.1145/116873.116880

2. Capin, T., Pulli, K., Akenine-Moller, T.: The state of the art in mobile graphics research. Computer Graphics and Applications, IEEE 28(4), 74-84 (2008). DOI 10.1109/MCG.2008.83

3. Gi, Y.J., Park, Y.S., Seo, S.H., Yoon, K.H.: Mosaic rendering using colored paper. In: Proceedings of the 7th International Conference on Virtual Reality, Archaeology and Intelligent Cultural Heritage, VAST'06, pp. 25-30. Eurographics Association, Aire-laVille, Switzerland, Switzerland (2006). DOI 10.2312/VAST/VAST06/025-030. URL http://dx.doi.org/10.2312/VAST/VAST06/025-030

4. Godwin-Jones, R.: Emerging technologies: Mobile apps for language learning. Language Learning \& Technology 15(2), 2-11 (2011)

5. Han, M., Kang, D., Yoon, K.: Efficient paper mosaic rendering on mobile devices based on position-based tiling. Journal of Real-Time Image Processing 9(3), 549-556 (2014). DOI 10.1007/s11554-013-0371-0. URL http://dx.doi.org/10.1007/s11554-013-0371-0

6. Hoff III, K.E., Keyser, J., Lin, M., Manocha, D., Culver, T.: Fast computation of generalized voronoi diagrams using graphics hardware. In: Proceedings of the 26th Annual Conference on Computer Graphics and Interactive Techniques, SIGGRAPH '99, pp. 277-286. ACM Press/Addison-Wesley Publishing Co., New York, NY, USA (1999). DOI 10.1145/311535.311567. URL http://dx.doi.org/10.1145/311535.311567

7. Jacott, L.: Mobile learning. a handbook for educators and trainers - edited by agnes kukulska-hulme and john traxler. British Journal of Educational Studies 57(3), 337-339 (2009)

8. Kang, D., Seo, S., Ryoo, S., Yoon, K.: A study on stackable mosaic generation for mobile devices. Multimedia Tools and Applications 63(1), 145-159 (2013). DOI 10.1007/s11042-012-1065-5. URL http://dx.doi.org/10.1007/s11042-012-1065-5

9. Kang, D., Tian, F., Sahandi, R.: Educational folktale e-book with collage illustratable tool. In: International Conference on Technologies for E-Learning and Digital Entertainment, pp. 15-26. Springer (2016)

10. Lehner, F., Nösekabel, H.: The role of mobile devices in e-learning - first experiences with a wireless e-learning environment. In: Proceedings IEEE International Workshop on Wireless and Mobile Technologies in Education, WMTE '02, pp. 103-106. IEEE Computer Society, Washington, DC, USA (2002). URL http://dl.acm.org/citation.cfm?id=645964.674255

11. Lloyd, S.P.: Least squares quantization in pcm. IEEE Transactions on Information Theory 28(2), 129-137 (2006). DOI 10.1109/TIT.1982.1056489. URL http://dx.doi.org/10.1109/TIT.1982.1056489

12. Mori, K., Ballagas, R., Revelle, G., Raffle, H., Horii, H., Spasojevic, M.: Interactive rich reading: Enhanced book reading experience with a conversational agent. In: Proceedings of the 19th ACM International Conference on Multimedia, MM '11, pp. 825-826. ACM, New York, NY, USA (2011). DOI 10.1145/2072298.2072478. URL http://doi.acm.org/10.1145/2072298.2072478

13. Munshi, A., Ginsburg, D., Shreiner, D.: OpenGL ES 2.0 programming guide. Pearson Education (2008) 
14. Seo, S., Park, Y., Kim, S., Yoon, K.: Colored-paper mosaic rendering. In: Proceedings of the 28th Annual Conference on Computer Graphics and Interactive Techniques, SIGGRAPH '01 Sketches \& Applications, p. 157. ACM, New York, NY, USA (2001) 


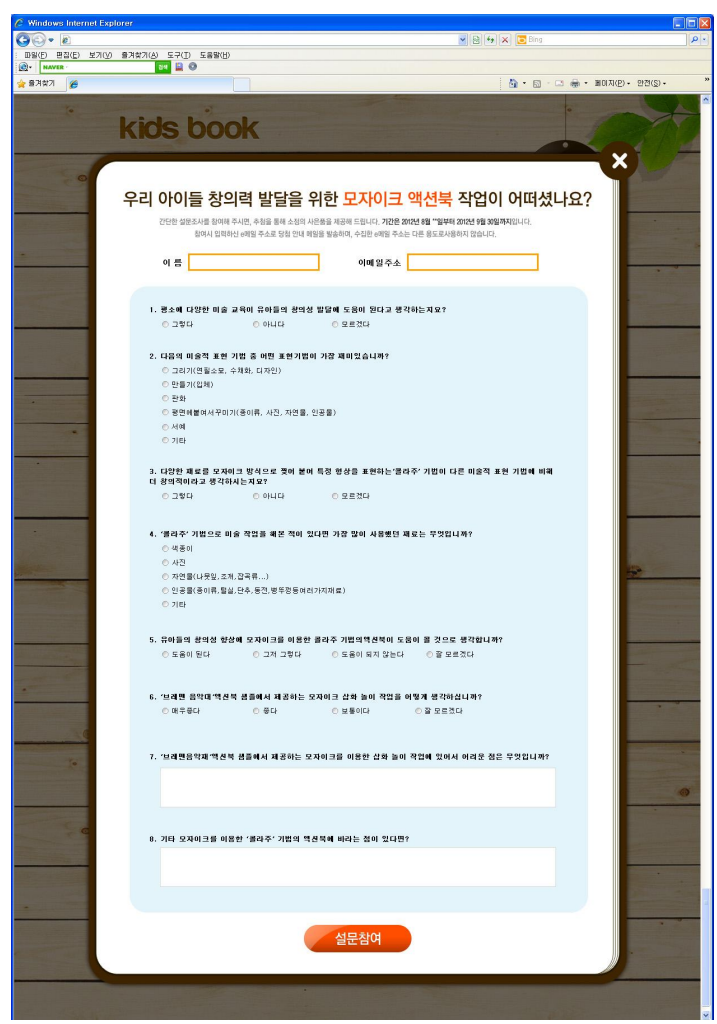

(a) Survey form

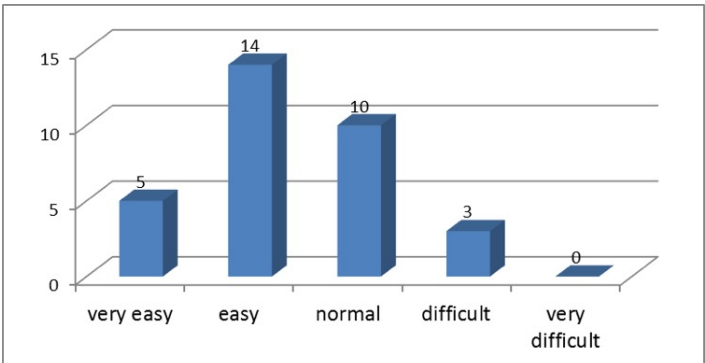

(b) Results on usability

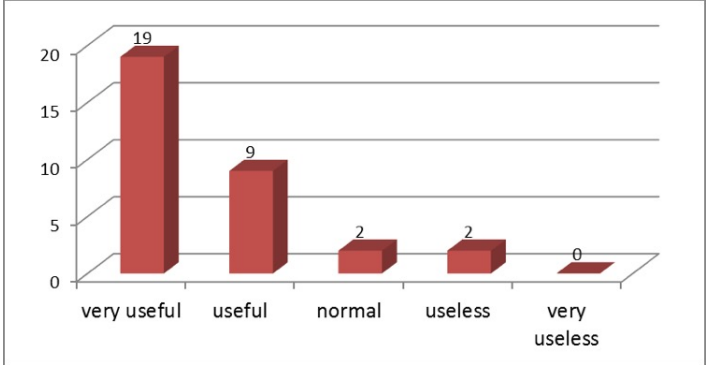

(c) Results on usefulness

Fig. 13 Evaluation results of a survey on usability and usefulness. 